\title{
Stochastic Geometry Based Framework for Coverage and Rate in Heterogeneous Networks with Sectored Fractional Frequency Reuse
}

\author{
Sani Umar Abdullahi*, Jian Liu, Seyed Alireza Mohadeskasaei \\ Department of Computer and Communication Engineering, University of Science and Technology Beijing (USTB), Beijing, P. R. China \\ Email address: \\ Saniumar.a.ng@ieee.org (S. U. Abdullahi), liujian@ustb.edu.cn (Jian Liu), alireza.kasaee@gmail.com (S. A. Mohadeskasaei) \\ ${ }^{*}$ Corresponding author
}

To cite this article:

Sani Umar Abdullahi, Jian Liu, Seyed Alireza Mohadeskasaei. Stochastic Geometry Based Framework for Coverage and Rate in Heterogeneous Networks with Sectored Fractional Frequency Reuse. American Journal of Networks and Communications. Vol. 6, No. 1, 2017, pp. 20-34. doi: 10.11648/j.ajnc.20170601.12

Received: March 9, 2017; Accepted: March 18, 2017; Published: April 3, 2017

\begin{abstract}
Modern day cellular networks are driven by the need to provide ubiquitous connectivity with very high spectral efficiency to both indoor and outdoor users, hence the need to deploy small cells over conventional macrocells in a Heterogeneous Network (Hetnet) deployment. To alleviate the resulting inter-cell and cross-tier interference, effective intercell interference coordination (ICIC) schemes such as Fractional Frequency Reuse (FFR) are employed, and have been widely studied in perfect geometry network scenarios which are too idealistic and not easily adaptable to the complexity of Hetnets. This work provides an analytical framework for the performance of such FFR schemes in Hetnets with antenna sectorization employed at the macro tier, by leveraging stochastic geometry tools to model base station locations of both macro and femto tiers using the Poisson Point Process (PPP). We study the effects of varying system parameters and consider cross-tier femto interference commonly ignored in many analytical works in literature. Furthermore, the femtocells employ a sensing algorithm to minimize spectrum sharing with macro users in close proximity, especially at the transition areas of center and edge region where cross-tier interference could be monumental. Numerical simulations are used to evaluate performance of the proposed framework in terms of coverage probability and average user rate, and results are compared with traditional FFR schemes and the No-FFR deployment. To the best of the author's knowledge, this is the first analytical framework characterizing sectoredFFR schemes using stochastic geometry tools in Hetnets.
\end{abstract}

Keywords: Heterogeneous Networks, Intercell Interference Coordination, Fractional Frequency Reuse, Stochastic Geometry, Poisson Point Process, Coverage, Rate

\section{Introduction}

Over the past few years, the mobile communications industry has experienced unprecedented explosion in data demand by advent of newer, smarter devices and systems, requiring ubiquitous connectivity and seamless quality of experience. Traditional capacity enhancing methods in macro-centric deployments such as increasing spectrum, cell splitting, improved modulation and other physical layer improvements are not sufficient, and hence a Heterogeneous network (Hetnet) architecture has been proposed by the $3^{\text {rd }}$ Generation Partnership Project (3GPP) which consists of diverse network tiers with varying transmit powers, densities and coverage areas - macrocells, picocells/relays, femtocells and/or distributed antenna systems [1]. The resulting Hetnet thus provides improved spatial reuse for higher network capacity, improved coverage, and better energy efficiency with significantly reduced cost. For indoor coverage, femtocells are particularly important to enhance degraded macro tier penetration and capacity especially since close to $70 \%$ of all data traffic has been found to originate indoors [2]. Femtocells are plug-and-play type access points with low-power (10-100mw) and short-range (10-30m) operating within licensed spectrum of operators often deployed in unplanned manner by end-users either in co-channel mode to macrocells for better spectrum utilization [3-4] or in 
orthogonal mode for avoiding cross-tier interference at cost of poor spectral efficiency [5]. Many femtocells restrict access to only authorized subscribers for economic or security reasons and are said to belong to a closed subscriber group (CSG).

Despite the numerous benefits of heterogeneous networks, the major challenge is of inter-cell and cross-tier interference since intracell interference is avoided inherently by Orthogonal Frequency Division Multiple Access (OFDMA) features. To alleviate the inter-cell and cross-tier interference capable of degrading performance of UEs at the boundary of cells or close to restricted access points under CSG mode, an inter-cell interference coordination (ICIC) scheme called Fractional Frequency Reuse (FFR) is used which is very effective for OFDMA-based systems such as Long Term Evolution (LTE) and LTE-Advanced (LTE-A) due to its low implementation complexity, minimal signaling overhead and significant performance gains [6]. Monte Carlo simulations have been used successfully in modeling single-tier networks for performance evaluation whereby every simulation is based on the specific scenario of interest and selected deployment parameters. However in Hetnets, the increasing deployment parameters of varying network tiers make it extremely exhaustive and challenging to investigate all possible scenarios and adequately analyze the spatial signalto-interference-plus-noise (SINR) distribution in finite time, and hence the need for newer, tractable models that can adequately relate performance metrics with key network parameters without the need for case-specific, extensive and complex simulations [7-9].

Recently, tools of stochastic geometry using Point Processes (PP) have been used to capture the non-uniform deployment in Hetnets by averaging over the entire cell coverage area over many spatial realizations [9-14]. By assuming base station locations of the different tiers to be given by some certain PP, the SINR distribution of a typically random UE in the plane can be accurately analyzed with minimal numerical complexity and relying only on key network parameters. The Poisson Point Process which assumes complete randomness is the simplest, most popular and most tractable PP and even though PPP-based models are ideally suited for femtocells due to ad-hoc deployments by consumers, recent studies have shown that they are as accurate even for macrocells as traditional grid models when compared to real measurements of actual $4 \mathrm{G}$ networks in both homogeneous [9] and heterogeneous deployments [10]. Importantly, stochastic geometry-based models are able to provide useful, analytical insights to design performance metrics and trade-offs in any configuration using mathematical tools unlike the grid models [7], [14].

\subsection{Fractional Frequency Reuse (FFR)}

The basic idea of FFR is to partition the entire spectrum into multiple sub-bands, such that UEs located close to the MeNB with good received signal quality utilize fullfrequency reuse on a certain frequency partition, while those with poor signal quality located at the boundary region of the cell are served with higher reuse factors on other sub-bands to minimize co-channel interference [4]. By so doing, the use of FFR in mobile networks leads to natural tradeoffs between achieving high spectrum utilization with full-frequency reuse systems (FR-1) and improved coverage for edge UEs in frequency reuse-n systems (e.g. FR-3). The main FFR deployment modes are: Strict FFR, Soft FFR and SectoredFFR (FFR-3, FFR-6 etc.), all of which are shown in Figure 1 for a hexagonal deployment. Strict FFR is the base-line FFR where the center region utilizes a common sub-band across all cells with full-frequency reuse (FR-1), while the bandwidth of the edge region is divided across the cells based on a frequency reuse factor $\Delta$ such that a total of $\Delta+1$ subbands are required. In Soft FFR, the edge region employs same bandwidth partitioning as Strict FFR, but the center region can now share edge sub-bands of neighbouring cells improving the bandwidth efficiency since all the $\Delta$ subbands are available in each cell, albeit at the cost of increased interference [6]. Soft FFR therefore employs power control to improve performance of edge UEs. Sectored FFR is an improvement of Strict FFR where directional antennas are employed by the MeNB to further minimize co-channel interference and also improve spectrum efficiency since all available $\Delta+1$ sub-bands are used in each cell. By focusing energy in the desired direction only, received signal quality to intended UEs is significantly increased without the need for complex power control as in Soft FFR.

\subsection{Related Work and Contributions}

There have been a lot of contributions in the literature on use of FFR as an efficient ICIC technique in multi-tier OFDMA networks in both static and dynamic operation modes based on regular grid models relying almost entirely on extensive simulations. Dynamic schemes [15-17] exhibit more resilience to traffic and interference variation but also have higher signaling overheads and increased complexity, making the static schemes [6, 18-21] very popular when appropriately provisioned. Such regular grid models make idealistic assumptions and are not practical for realistic network deployments with irregular geometry and complex distributions of diverse access points as obtainable in Hetnets. As stated earlier, stochastic geometry models are well-placed to analyze such networks accurately, but employing ICIC measures, especially FFR has proven very challenging due to the difficulty in spatially partitioning the irregular cells into center and edge regions, and more importantly, due to correlations that may occur between base stations sharing same sub-band which would violate the independence property of the PPP [14]. One of the first existing works employing stochastic geometry tools in FFRaided networks are [22-23] where authors were able to overcome the above challenges by using an SINR threshold instead of spatial distance to partition cell center and cell edge users, and proposing a worst-case FFR where edge subbands are allocated randomly to preserve the PPP property.

This work was extended in [24-25] for a two-tier network and performance was quantified in terms of coverage 
probability and average rate. Authors in [26] employ FFR in networks with imperfect grid by abstracting base station locations as perturbed from their ideal hexagonal lattice positions and used Monte Carlo simulations for performance evaluation. All the existing analytical works so far investigating FFR-based multi-tier networks have focused mainly on omni-directional cases, and analyzed Strict or Soft FFR. The recent work in [27] did propose a sectored model with FFR and used PPP and HCPP for modeling base station locations but resorted to use of Monte Carlo simulations and not analytical evaluation, hence could not provide tractable insights into design guidelines and performance.

In this work, we provide an analytical framework for performance evaluation of a two-tier OFDMA network using sectored-FFR for co-tier and cross-tier interference management under closed access by leveraging tools of stochastic geometry. Two independent spatial PPPs are used to model base station distributions of the different tiers, and average SINR is employed to classify MUEs as center or

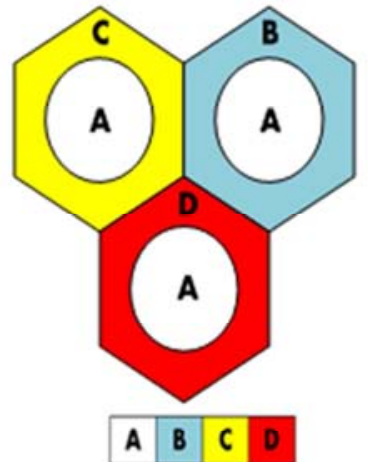

(a) Strict FFR

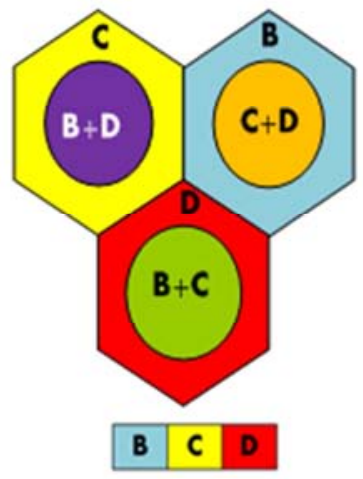

(b) Soft FFR edge UEs respectively. The femtocells execute a sensing algorithm to avoid use of same sub-bands as MUEs in the same area, and also ensure that same spectrum resources are not shared with MUEs at the transition areas of center and edge region respectively, so as to minimize severe cross-tier interference at that region commonly ignored in most previous works. The derived SINR distribution framework is verified by numerical simulation and then results compared with other FFR schemes and the No-FFR system. Lastly, average network spectral efficiency is computed for the case of both 3-sectored and 6-sectored FFR deployments to identify the effects of interference reduction via use of directional antennas under the analytical model. To the best of the authors' knowledge, this is the first contribution in the literature that provides insights into FFR-aided OFDMA heterogeneous networks employing directional antennas using real analytical tools from stochastic geometry for modeling all the tiers as well as evaluate the performance metrics.

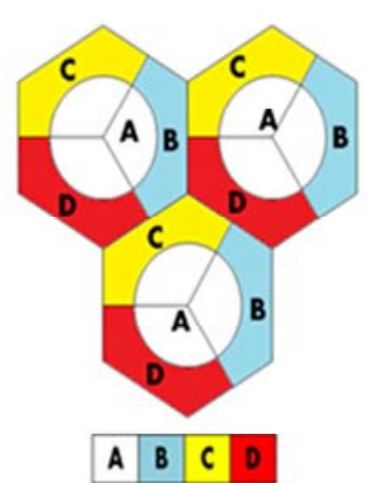

(c) FFR-3

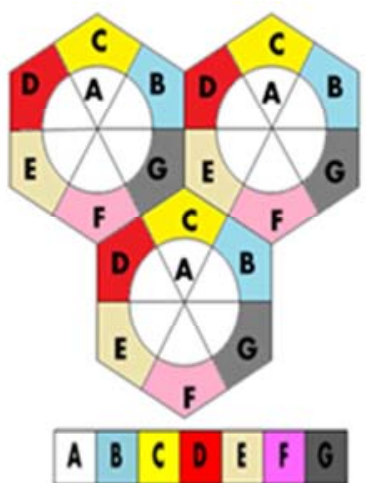

(d) FFR-6

Figure 1. Different FFR variants under hexagonal grid model.

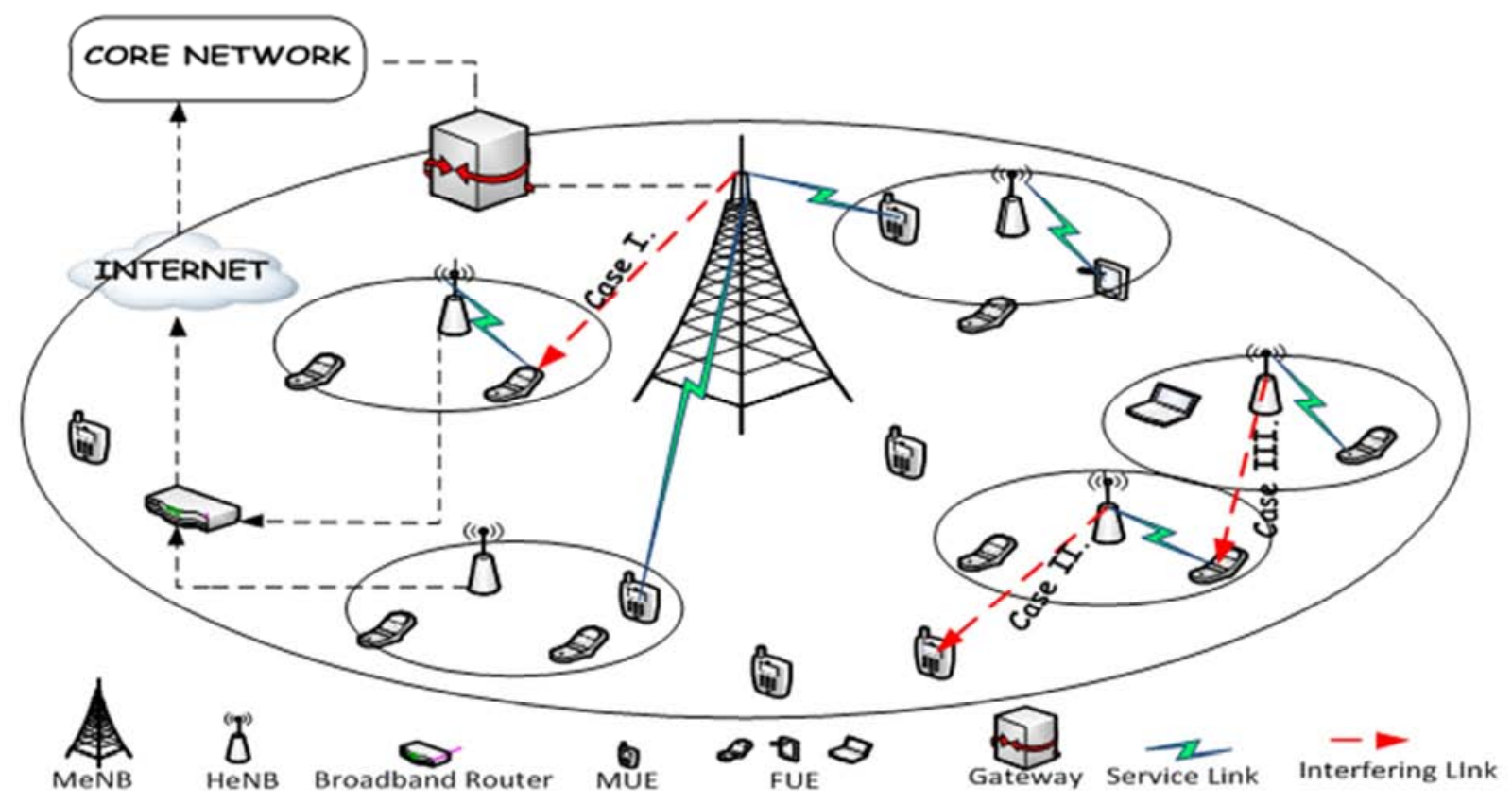

Figure 2. Two-Tier heterogeneous cellular network showing downlink interferences. 


\section{System Architecture and Problem Formulation}

\subsection{Two-Tier Macro-Femto Network Model}

Figure 2 shows the architecture of a typical two-tier heterogeneous network where macrocells are overlaid with femtocells and the downlink interference scenarios are given as; Case I - interference from Macro base station (MeNB) to femto user equipments (FUEs), Case II - interference from femto access points (HeNB) to macro user equipments (MUEs), and lastly Case III - interference from one HeNB to other FUEs of different CSGs. In this work, the macro and femto base stations are spatially distributed as independent homogeneous PPPs $\Phi_{m}$ and $\Phi_{f}$ with intensity $\lambda_{m}$ and $\lambda_{f}$ respectively. The resulting coverage topology is shown in Figure 3 where the inverted triangles represent the MeNBs with their respective coverage areas, and the femtocells are denoted by small circles with yellow coverage areas, while the MUEs are uniformly distributed over the entire coverage area as black dots. Directional antennas are employed by the MeNBs in the edge region, and average SINR threshold $\Gamma_{\text {MACRO }}$ is used to partition MUEs into cell center and cell edge region respectively. FUEs also constitute uniform distribution within their associated HeNBs, where the femtocells operate in closed access mode and by avoiding spectrum sharing with MUEs at boundary of cell center and cell edge region through a sensing algorithm, a separate virtual threshold $\Gamma_{\text {FEMTO }}$ is assumed to classify FUEs into center and edge respectively different than $\Gamma_{\text {MACRO }}$.



Figure 3. PPP-distributed Two-Tier Cellular Network Coverage Region.

Without loss of generality, we assume the typical user to be located at the origin due to Slivnyak's theorem which states that the statistics seen from a PPP is independent of the test location [8], [14]. The standard path loss propagation model is employed with path loss exponent $\alpha>2$ and the noise is additive and constant represented as $\sigma^{2}$. Rayleigh fading with unit mean is assumed for the small scale fading between the considered typical user and both the serving and interfering base stations for improved tractability in the analysis (i. e. $h \approx \exp (1)$ and $g \approx \exp (1)$ ). For the macro tier utilizing directional antennas at the edge, we can use the tuple $\left\{\lambda_{m}, P_{m}, T_{m}, G_{m}\{\}.\right\}$ to represent its properties whereby $G_{k}\{$.$\} is the antenna gain pattern function for \Delta$ edge subbands randomly allocated to $\Delta$ edge sectors for a frequency reuse factor of $\Delta$ at the edge. For any random edge sub-band $\delta \in\{1,2, \ldots . \Delta\}$, the directional antenna at location $x$ has a sector angle $\theta_{\delta}(x)$ which is defined from the bore-sight of the antenna to the positive real axis. $G_{k}\left(\psi_{x}\right)$ is therefore the directional antenna gain in the direction of the typical UE at origin, whereby $\psi_{x}$ is given as;

$$
\psi_{x} \triangleq\left(\angle x-\theta_{\delta}(x)\right) \quad \bmod 2 \pi
$$

$\angle x$ is the argument of $x$ and the resultant angle $\left(\angle x-\theta_{\delta}(x)\right)$ is adjusted within the range $[0,2 \pi)$. Based on the pattern of the directional antenna, the maximum directional antenna gain from all $\Delta$ sectors at location $x$ towards the typical UE is defined as $G_{k, \max }\left(\psi_{1}(x)\right)$ expressed as;

$$
\begin{aligned}
G_{m, \max }\left(\psi_{1}(x)\right) & \triangleq \max _{i \in\left\{1,2, \ldots, \Delta_{k}\right\}} G_{m}\left(\psi_{i}(x)\right) \\
& =\max _{i \in\left\{1,2, \ldots, \Delta_{m}\right\}} G_{m}\left(\left(\psi_{1}(x)-\frac{2 \pi(i-1)}{\Delta_{k}}\right) \bmod 2 \pi\right)
\end{aligned}
$$

An illustration of a typical irregular cell with the described configuration and employing $120^{\circ}$ sectoring at the macrocell is shown in Figure 4 where all spatial partitions are imaginary and only shown for ease of explanation and analytical convenience.

\subsection{Cell Association and SINR Model}

Similar to [12], the typical UE is hereby assumed to connect to the base station with maximum equivalent received power which is based on an equivalent received power connectivity (ERPC) model ideal for heterogeneous networks in both closed and open access operation mode since it averages out fading fluctuations and also considers transmit powers of the different tiers as well as long-term path-loss. It is also easily applicable for the sectored antenna scenario as employed in this work and also shown in [28]. Aside being more suitable to heterogeneous network deployment, this model is more practical since it reflects realistic UE cell selection processes in modern mobile networks based on pilot measurements that average out fastfading and frequency selective fading rather than relying on instantaneous SINR values. The long-term received power from the MeNB at location $x$ is therefore defined as $P_{m} G_{m, \max }\left(\psi_{1}(r)\right)\left\|x_{m}\right\|^{-\alpha}$, and the UE associates with the sector of the MeNB with maximum long-term received power where the selected base station location is thereby given as; 


$$
\varphi=\arg \max _{x \in \Phi_{m}}\left[P_{m} G_{m, \max }\left(\psi_{1}(r)\right)\left\|x_{m}\right\|^{-\alpha}\right]
$$

We can thus define a random variable;

$$
\widetilde{P}_{\max }=P_{m} G_{m, \max }\left(\psi_{1}(\varphi)\right)\left\|\varphi_{m}\right\|^{-\alpha}
$$

to represent the maximum long-term received power from all base stations (and all sectors). It can be seen that this cell association model easily reverts to the voronoi tessellation model where the closest base station is selected for the case of a single tier network or when the powers of all the tiers are equal, and omnidirectional antennas are employed (i.e. $\left.G_{m, \max }\left(\psi_{1}(r)\right)=1\right)$.

Based on the above model, it implies that the equivalent received power of all other base stations must be less than the selected base station using maximum ERP. Therefore the SINR in the downlink is given in (5) where $G_{m, \max }\left(\psi_{1}(r)\right)=1$ for center region MUEs.

$$
\operatorname{SINR}=\frac{P_{m} G_{m, \max }\left(\psi_{1}(\varphi)\right) \hat{h_{m}} \varphi_{m}^{-\alpha}}{\sigma^{2}+I_{m}+I_{f}}
$$

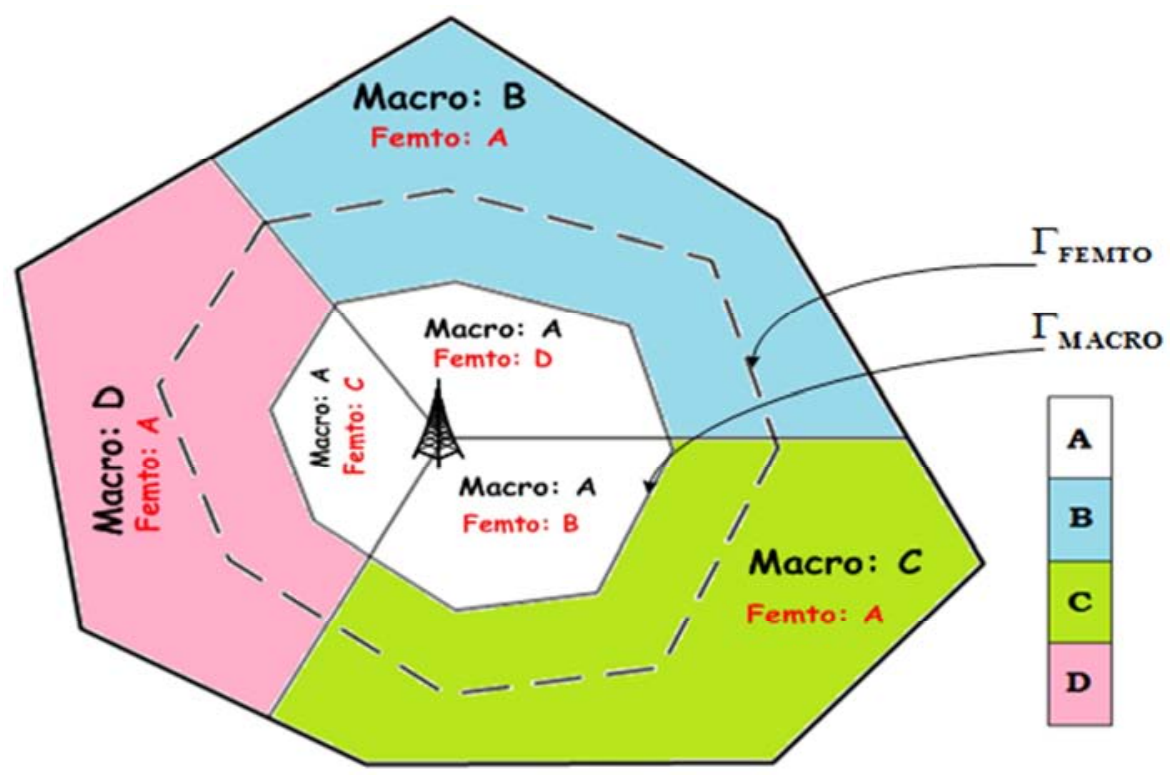

Figure 4. Virtual model of typical irregular cell with tri-sectored-FFR.

$I_{m}$ is the co-tier interference from macrocells. For center UEs, it is given as $I_{m}^{C R}=\sum_{y \in \Phi_{m} \backslash\{\varphi\}} P_{y} g_{x}\|x\|^{-\alpha}$ which contains interference from all macrocells in the network except the associated MeNB $\varphi$ since the common sub-band is shared by all MeNBs in FFR using omnidirectional antennas for center UEs, while for edge UEs, it is given as $I_{m}^{E R}=\sum_{y \in \Phi_{m} \backslash\left\{\varphi_{\}}\right\}} P_{y} G_{y}\left(\psi_{\delta}(x)\right) \hat{g_{x}}\|x\|^{-\alpha}$ where the interference is from only those macrocell sectors sharing same sub-band as reference UE. $I_{f}$ is the cross-tier interference from femtocells using same sub-band as the reference UE given as $I_{f}=\sum_{y \in \Phi_{f}} P_{y} g_{x}\|x\|^{-\alpha}$.

\subsection{Femtocell Resource Allocation}

In order to minimize cross-tier interference, the femtocells employ Algorithm 1 to avoid co-channel spectrum usage with MUEs in close proximity. The key steps are hereby presented with comments and the operational principles detailed afterwards.

Table 1. Algorithm for femtocell spectrum allocation.

\begin{tabular}{lll}
\hline \multicolumn{2}{l}{ Algorithm for Femtocell Spectrum Usage } \\
\hline Input: & $S B$ & \% Set of all available frequency resources in a MeNB \\
Output: & $S B_{u}$ & \% Usable frequency sub-bands for any HeNB \\
1: & For all HeNBs $f \in \mathrm{F}$ & \\
$2:$ & $S B_{u} \leftarrow S B$ & \\
$3:$ & End for & \\
$4:$ & For all HeNBs $f \in \mathrm{F}$ & \% Femtocells sense pilot signals from macro cells \\
$5:$ & $\Gamma \leftarrow$ set of RSSI values for all sub-bands $S B$ & \% Find the pilot signal with highest power \\
6: & $\Gamma_{S B}^{\max , 1} \leftarrow$ Highest RSSI value & \% Femtocell is in center region if sub-band A has highest Power \\
7: & If $\Gamma_{S B}^{\max , 1}==\Gamma_{A}$ &
\end{tabular}




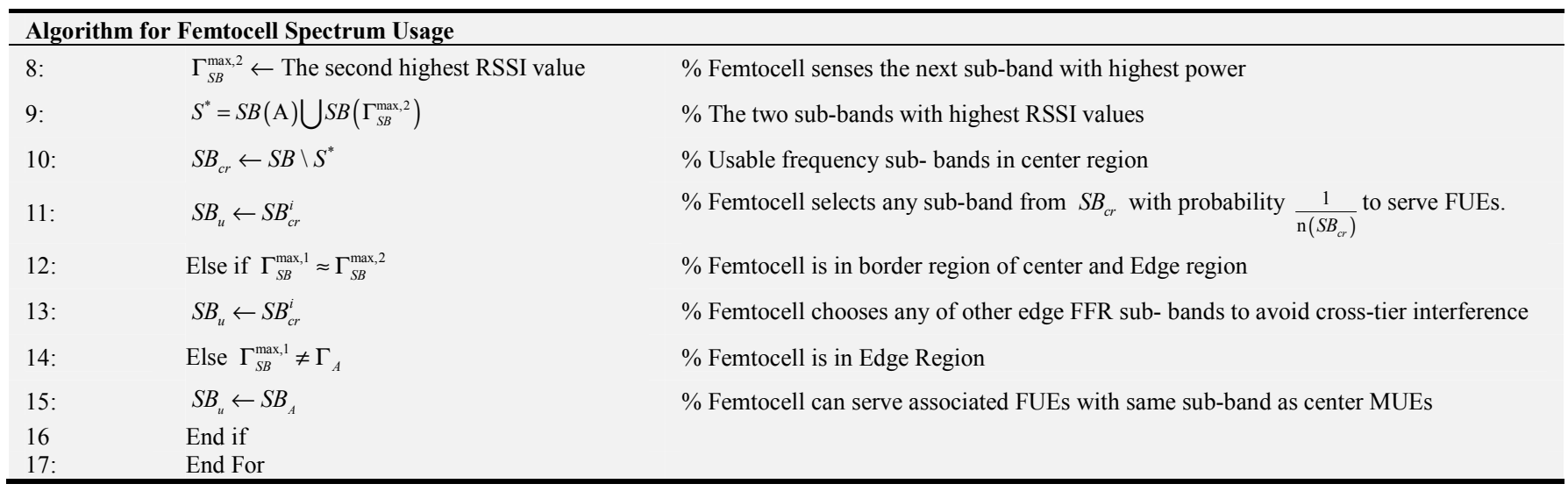

Building from [6], the set of usable spectrum resources for the femtocells $S B_{u}$ is initialized by the set of all available subbands in a macrocell ( $S B=\{\mathrm{A}, \mathrm{B}, \mathrm{C}, \mathrm{D}\}$ for an FFR-3 scheme as shown in Figure 2c.). Upon start-up, the femtocell $f$ senses all neighbouring macro pilot signals and determines the one with strongest received signal strength indicator (RSSI) labeled as $\Gamma_{S B}^{\max , 1}$. If this sub-band is the common subband shared by all MUEs in center region, then the femtocell identifies as a center femtocell and proceeds to determine the sub-band with the second highest RSSI labeled $\Gamma_{S B}^{\max , 2}$ and the sub-band is defined as $S B\left(\Gamma_{S B}^{\max , 2}\right)$ which is probably the sub-band the MeNB serves edge MUEs in this sector. Hence, the femtocell avoids these two sub-bands with relatively high RSSI, and chooses randomly to serve associated FUEs with any of other edge FFR sub-bands.

In step 12, when the femtocell recognizes that $\Gamma_{S B}^{\max , 1} \approx \Gamma_{S B}^{\max , 2}$, it identifies as being in boundary of center and edge region of the macrocell, and thereby avoids the common MUE sub-band (sub-band A) shared by all center MUEs since the cross-tier interference from MeNB would be quite significant. Our work in [21] has shown the degrading effects of such boundary cross-tier interference commonly ignored by many FFR works in literature. Finally, when the femtocell finds that $\Gamma_{S B}^{\mathrm{max}, 1}$ does not correspond to the common macrocell center sub-band, then femtocell identifies as in the edge region, and can safely reuse the common sub-band with center MUEs with relatively minimal cross-tier interference as proven in [6, 21-25].

\section{Coverage Probability Framework}

In this section, we use stochastic geometry tools to derive theoretical expressions of coverage probability for a two-tier macro-femto OFDMA network employing sectored FFR under closed access mode. Rayleigh fading is assumed for both serving and interfering links to the UE for more tractability. The coverage probability of a UE is determined when the instantaneous SINR level exceeds a certain threshold $T$. It is mathematically equivalent to the complementary cumulative distribution function (CCDF) of the SINR distribution and is given as; $\mathrm{CP}=\operatorname{Pr}(\operatorname{SINR}>T)$. Since the equivalent received power of all other interfering base stations must be less than that of the selected base station (i.e. $\widetilde{P}_{\max }$ ) using maximum ERP model, thus the cumulative distribution function (CDF) of the random variable $\widetilde{P}_{\text {max }}$ for the edge region where sectored are antennas are employed is defined as

$$
F_{\widetilde{P}_{\max }}(q)=\mathbb{P}\left(\widetilde{P}_{\max } \leq q\right)=\mathbb{P}\left(\widetilde{P}_{\max }=P_{m} G_{m, \text { max }}\left(\psi_{1}(r)\right)\left\|r_{m}\right\|^{-a} \leq q\right)
$$

which simplifies to (7) as proven in [28];

$$
F_{\vec{P}_{\max }}(q)=\left[\exp \left(-2 \pi \lambda_{m} \int_{0}\left(1-F_{G_{m_{\text {max }}}}\left(\frac{q\|r\|^{\alpha}}{P_{m}}\right)\right) r d r\right)\right]
$$

Where $G_{m, \max }(x)$ is a random variable when $x$ is randomly selected from the plane, and its CDF is denoted as $F_{G_{m, \text { max }}}($.$) .$ The probability density function (PDF) of $F_{\tilde{P}_{\max }}(q)$ is therefore found as its numerical derivative expressed as;

$$
f_{\tilde{P}_{\text {max }}}(q)=\frac{\mathrm{d}\left(F_{\tilde{P}_{\text {max }}}(q)\right)}{\mathrm{dq}}
$$

When an omni-directional antenna is used, the gain is uniform in all directions and hence, $G_{m, \max }(\psi)=G_{m}(\psi)=1$ and so the CDF and PDF of $\widetilde{P}_{\max }$ become;

$$
\begin{aligned}
& F_{\tilde{P}_{\max }}(q)=\left[\exp \left(-2 \pi \lambda_{m} \int_{0}^{\left(P_{m} / q\right)^{1 / \alpha}} r \mathrm{~d} r\right)\right]=\exp \left(-\pi \lambda_{m}\left(\frac{P_{m}}{q}\right)^{2 / \alpha}\right) \\
& f_{\tilde{P}_{\max }}(q)=\frac{\mathrm{d} F_{\tilde{P}_{\max }}(q)}{\mathrm{dq}}=\frac{2 \pi \lambda_{k} P_{k}^{2 / \alpha}}{\alpha}\left(q^{-2 / \alpha^{-1}}\right) \exp \left(-\pi \lambda_{m} P_{m}^{2 / \alpha} q^{-2 / \alpha}\right)
\end{aligned}
$$

Note: Although [28] provides guidelines for coverage probability analysis of two-tier networks with directional antennas, it is very different from our contribution as it does not employ FFR and assumes open-access operation where the typical UE connects to either of the tiers, thereby neglecting cross-tier interference which can be very significant with dense femtocell deployment.

At this point, we first define the coverage probability for a typically random UE under closed access mode with no frequency reuse and no sectored antennas to be our base-line framework which is applicable for center MUEs in this work. 
This is a variant of the form given in [12] but with consideration to femtocell cross-tier interference under closed-access.

\subsection{Lemma 1}

Under the heterogeneous cellular network deployment with closed-access operation mode, omnidirectional antenna and no frequency reuse, the coverage probability of a typically randomly located macro tier UE is given by;

$$
\begin{aligned}
\mathrm{CP} & =\frac{2 \pi \lambda_{m} P_{m}^{2 / \alpha}}{\alpha} \int_{0}^{\infty} e^{\left(-\frac{\sigma^{2} T}{q}\right)} \cdot q^{-\frac{2}{\alpha}-1} \cdot \exp \left(-\frac{\pi}{q^{2 / \alpha}}\left[\lambda_{m} P_{m}^{2 / \alpha}(1+\rho(T, \alpha))\right]\right) . \\
& \exp \left(-\pi \lambda_{f}^{\wedge}\left(\frac{T}{q} P_{f}\right)^{\frac{2}{\alpha}} \frac{2 \pi}{\alpha \sin (2 \pi / \alpha)}\right) \mathrm{dq}
\end{aligned}
$$

Where $\rho(T, \alpha)=T^{2 / \alpha} \int_{T^{-2 / \alpha}}^{\infty} \frac{1}{1+u^{\alpha / 2}} \mathrm{~d} u$, Proof: Proof is given in Appendix A.

The first exponential term represents the noise term, the second exponential term represent co-tier macro interference while the cross-tier interference is represented by the third exponential term. Upon careful comparisons and replacing $\left(P_{m} / q\right)^{1 / \alpha}$ with $r$ in (10), the result is found to be exactly the same as the popular coverage probability for a typical UE under Rayleigh fading derived in [9] and subsequent works but only that (10) includes cross-tier interference from femtocells.

Next, we extend this framework for the case of directional antennas building from the theory in [29] and similar to [28] which can be applied for edge UEs in our FFR model as will be shown in later sections.

\subsection{Lemma 2}

Considering directional antennas are employed with different frequencies per sector, the coverage probability of a typically randomly located UE in a heterogeneous network under closed access is given by;

$$
\begin{aligned}
\mathrm{CP} & =\int_{0}^{\infty} \exp \left(-\frac{\sigma^{2} T}{q}\right) \exp \left(-\frac{\lambda_{m}}{2}\left(\frac{P_{m}}{q}\right)^{2 / \alpha} M_{k}(T, \alpha)\right) . \\
& \exp \left(-\pi \lambda_{f}^{\wedge}\left(\frac{T}{q} P_{f}\right)^{2 / \alpha} \frac{2 \pi}{\alpha \sin (2 \pi / \alpha)}\right) f_{\tilde{P}_{\max }}(q) \mathrm{dq}
\end{aligned}
$$

Proof:

The proof tracks that of Lemma 1 up to (A2 (c)). Since the gain for directional antenna is non-uniform, hence the CDF and PDF will have unique values for each antenna pattern and therefore the expressions in (9) cannot be used in this case. The value of $\mathcal{L}_{I_{m}^{\prime}}\left(\frac{T}{q} \mid \widetilde{P}_{\max }\right)$ now depends on antenna pattern as well and is evaluated as; Step (a) is as a result of the i.i.d distribution of fading $\left\{\mathrm{g}_{x}^{\wedge}\right\}$. Also, $\left\{\mathrm{g}_{x}\right\}$ and $\psi_{\delta}(x)$ are independent, where $\psi_{\delta}(x)$ can also be assumed to be a random variable distributed uniformly within the interval $[0,2 \pi)$ so that the interfering macrocells will form a thinned PPP of density $\lambda_{m} \frac{\mathrm{d} \theta}{2 \pi}$. Hence, step (b) follows using the probability generating functional (PGFL) of the thinned PPP whereby the integration limits are from $\left(P_{m} G_{m, \max }(\theta) / \widetilde{P}_{\max }\right)^{1 / \alpha}$ to $\infty$ so as to represent the fact that the long-term received powers of all interfering base stations are positive and cannot be greater than $\widetilde{P}_{\max }$. Lastly, step (c) is derived due to Rayleigh fading assumption. Substituting for $s=\frac{T}{q}$ and using the condition $\widetilde{P}_{\max }=q$;

$$
\begin{aligned}
& \mathcal{L}_{I_{m}^{\wedge}}\left(s \mid \widetilde{P}_{\max }\right)=\mathbb{E}_{I_{m}^{\wedge}}\left[\exp \left(-s I_{m}^{\wedge} \mid \widetilde{P}_{\max }\right)\right] \\
& \stackrel{\text { (a) }}{=} \mathbb{E}_{\phi_{k}}\left[\prod_{\substack{x \in \phi_{m} \backslash\{\varphi\} \\
P_{m} G_{m, \max }\left(\psi_{1}(x)\right)\|x\|^{-\alpha}<\widetilde{P}_{\text {max }}}} \mathbb{E}_{h_{x}, \psi_{\delta}(x)}\left[\exp \left(-s P_{m} G_{m}\left(\psi_{1}(x)\right) \cdot \mathrm{g}_{x}^{\wedge}\|x\|^{-\alpha}\right)\right]\right] \\
& \stackrel{\operatorname{cox})}{=} \exp \left(-\int_{0}^{2 \pi} 2 \pi \int_{\left(P_{m} G_{m, \text { max }}(\theta) / q\right)^{\frac{1}{\alpha}}}^{\infty}\left(1-\mathcal{L}_{h_{x}^{\wedge}}\left(s P_{m} G_{m}(\theta) v^{-\alpha}\right)\right) v \mathrm{~d} v \lambda_{m} \frac{\mathrm{d} \theta}{2 \pi}\right) \\
& \text { (c) } \exp \left(-\lambda_{k} \int_{0}^{2 \pi} \int_{\left(P_{m} G_{m, \max }(\theta) / q\right)^{\frac{1}{\alpha}}}^{\infty}\left(1-\frac{1}{1+s P_{m} G_{m}(\theta) v^{-\alpha}}\right) v \mathrm{~d} v \mathrm{~d} \theta\right)
\end{aligned}
$$




$$
\begin{aligned}
\mathcal{L}_{I_{m}}\left(\frac{T}{q} \mid \widetilde{P}_{\max }\right) & =\exp \left(-\lambda_{k} \int_{0}^{2 \pi} \int_{\left(P_{m} G_{m}, \max (\theta) / q\right)^{1 / \alpha}}\left(1-\frac{1}{1+\frac{T}{q} P_{m} G_{m}(\theta) v^{-\alpha}}\right) v \mathrm{~d} v \mathrm{~d} \theta\right) \\
& =\exp \left(-\frac{\lambda_{m}}{2}\left(\frac{P_{m}}{q}\right)^{2 / \alpha} \int_{0}^{2 \pi}\left(G_{m, \text { max }}(\theta)\right)^{2 / \alpha} \rho\left(\frac{T G_{m}(\theta)}{G_{m, \text { max }}(\theta)}, \alpha\right) \mathrm{d} \theta\right) \\
\mathcal{L}_{I_{m}^{\wedge}}\left(\frac{T}{q} \mid \widetilde{P}_{\max }\right) & =\exp \left(-\frac{\lambda_{m}}{2}\left(\frac{P_{m}}{q}\right)^{2 / \alpha} M_{m}(T, \alpha)\right) \\
\text { where } M_{k}(T, \alpha) & =\int_{0}^{2 \pi}\left(G_{m, \text { max }}(\theta)\right)^{2 / \alpha} \rho\left(\frac{T G_{m}(\theta)}{G_{m, \text { max }}(\theta)}, \alpha\right) \mathrm{d} \theta
\end{aligned}
$$

\subsection{Lemma 3}

(Sectored FFR, Edge UE closed access): Under the heterogeneous cellular network deployment with closedaccess operation mode employing sectored FFR, the coverage probability of a typically randomly located edge macro tier UE is given by;

$$
\begin{aligned}
& \mathrm{CP}(k, T)_{\{F F R-n\}}=\frac{\mathrm{CP}_{\left(\left\{\lambda_{m}\right\},\left\{P_{m}\right\},\left\{T_{m}\right\},\left\{G_{m}(.)\right\}\right)}}{1-\mathrm{CP}_{\mathrm{cl} .\left\{\left\{\lambda_{m}\right\},\left\{P_{m}\right\},\left\{T_{0}\right\}, 1\right)}}-\frac{\int_{0}^{\infty} \mathcal{Z}(k, T) \cdot f_{\tilde{P}_{\max }}(q) \mathrm{dq}}{1-\mathrm{CP}_{\mathrm{cl} .\left\{\left\{\lambda_{m}\right\},\left\{P_{m}\right\},\left\{T_{0}\right\}, 1\right)}} \\
& \text { Where } \mathcal{Z}(k, T)=\left[\begin{array}{l}
\left.\exp \left(-\frac{\sigma^{2}}{q}\left(T+T_{0}\right)\right) \cdot \exp \left(-\lambda_{m}\left(\frac{P_{m}}{q}\right)^{2 / \alpha}\left(\frac{1}{2} M_{m}(T, \alpha)+\pi \rho\left(T_{0}, \alpha\right)\right)\right) \times\right] \\
\exp \left(-\pi\left(\frac{P_{f}}{q}\right)^{2 / \alpha} \frac{2 \pi}{\alpha \sin (2 \pi / \alpha)}\left(T^{2 / \alpha} \lambda_{f}+T_{0}^{2 / \alpha} \lambda_{f}\right)\right)
\end{array}\right]
\end{aligned}
$$

Proof: The proof is given in Appendix B.

This coverage probability framework is for those UEs whose instantaneous SINR levels are less than the FFR threshold $T_{0}$ and are therefore classified as edge UEs who are served by one of the edge FFR sub-bands by any of the directional antennas employed in the edge region. Such UEs will experience new fading $\hat{g_{m}}$ and new co-tier and cross-tier interferences. We see from Figures 2 (c and d) that an edge MUE is interfered by macrocells and femtocells sharing the same sub-band and for macrocells, the direction of the interfering cells is important, hence we define the thinned density of interfering macrocells as $\lambda_{m} \frac{\mathrm{d} \theta}{2 \pi}$. For interfering femtocells $t$ edge UE, these will be center femtocells sharing same edge sub-band as the UE and can be approximated using the $\mathrm{CCDF}$ at $T_{0}$ to derive the thinned density as $\lambda_{f}^{\wedge}=\mathrm{CP}\left(T_{0}\right) \cdot \frac{\lambda_{f}}{\Delta} \quad$, where $\mathrm{CP}\left(T_{0}\right) \cdot \lambda_{j}$ represents center femtocells and is divided by number of edge FFR sub-bands to find the probability of interfering femtocells using same edge sub-band as typical edge UE.

\subsection{Lemma 4}

(Sectored FFR, Center UE closed access): For a typical UE under closed access mode in the downlink of a heterogeneous cellular network, the coverage probability for a center UE is given as;

$$
\mathrm{CP}(k, T)_{\mathrm{CR}}=\mathbb{P}\left(\frac{\frac{P_{m} g_{m} r_{m}^{-\alpha}}{\sigma^{2}+I_{m}+I_{f}^{v}}>\max \left\{T, T_{0}\right\}}{\frac{P_{m} h_{m} r_{m}^{-\alpha}}{\sigma^{2}+I_{m}+I_{f}}>T_{0}}\right)=\frac{\mathrm{CP}\left(\max \left\{T, T_{0}\right\}, \lambda, \alpha, 1\right)}{\mathrm{CP}(T, \lambda, \alpha, 1)}
$$

Proof: The UE will have SINR greater than the FFR threshold, and hence be served on the common sub-band with omni-directional antenna. Macro tier interference would still be from all other neighbouring macrocells, while femto tier interference would be from edge femtocells with thinned intensity approximated as $\lambda_{f}^{v}=\left\lfloor 1-C P\left(T_{0}\right)\right\rfloor \lambda_{f}$, where $\left\lfloor 1-C P\left(T_{0}\right)\right\rfloor$ is used to analytically represent edge region since the concept of spatial distances for classifying center and edge is not justifiable for an irregular, PPP-based topology. This bracket $\lfloor x\rfloor$ represents the floor function so that only integer values are obtained which are less than or equal to the actual value of $x$. Aside the inclusion of femto interference in our framework in (16), the result is almost identical to that derived in [23] for center UEs under strict FFR since the setup is same for both schemes in center region as shown in Figure 2.

\section{Average Rate and Network Spectral Efficiency}

In this section we use the derived SINR distribution framework to obtain analytical expressions for average rate of a typically randomly located UE under sectored FFR in the downlink of the presented heterogeneous network. Adaptive modulation and coding are assumed and like previous sections, exponential interference is employed. The results build from the key work presented in [9] which is the foundation for many recent analytical works using stochastic geometry in heterogeneous cellular networks. Subsequently, the total network sum rate is evaluated under varying femtocell deployments.

The average rate of a typical UE averaged over the base station locations and fading distribution is defined in (17) and expressed in units of nats $/ \mathrm{Hz}$ as given in [9] where it was explained that $1 \mathrm{bit}=\log _{e}(2)$ nats;

$$
\overline{\mathcal{T}}=\mathbb{E}[\ln (1+\mathrm{SINR})]=\int_{q>0} \mathbb{E}[\ln (1+\operatorname{SINR})] \cdot f_{\widetilde{P}_{\max }}(q) \mathrm{dq}
$$

\subsection{Lemma 5}

(Sectored-FFR, Edge UE): The average rate of a typical edge UE in the proposed model using sectored FFR in the downlink is given as; 


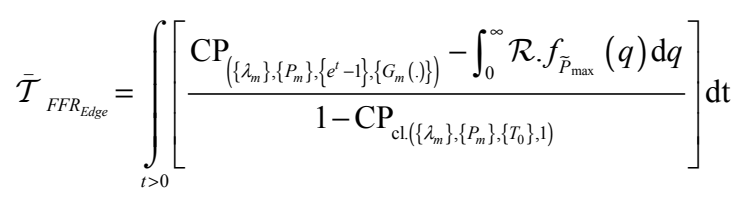

where $\mathcal{R}=\int_{0}^{\infty}\left[\exp \left(-\frac{\sigma^{2}}{q}\left(e^{t}-1+T_{0}\right)\right) \cdot \mathcal{L}_{I_{m}, I_{m}}\left(\hat{s_{m}}, s_{m} \mid \widetilde{P}_{\max }\right) \cdot \mathcal{L}_{I_{f}, I_{f}}\left(\hat{s_{f}}, s_{f} \mid \widetilde{P}_{\max }\right)\right]$ And $\hat{s_{m / f}}=\frac{e^{t}-1}{q}$ and $s_{m / f}=\frac{T_{0}}{q}$ Proof: The proof is provided in Appendix C.

The average for center UEs can be found similarly using the coverage probability results from Lemma 4 and so is excluded here for brevity.

In such PPP-based heterogeneous OFDMA networks, the fraction of resource blocks allocated to the edge region can be found using the CCDF evaluated at $T_{0}$ rather than the commonly used squared ratio of inner to edge region in grid based topologies.

$$
N_{\text {Edge }}^{\mathrm{RB}}=\left\lfloor 1-\mathrm{CP}\left(T_{0}\right) \cdot N_{\text {Total }}^{\mathrm{RB}}\right\rfloor
$$

The center resource blocks will therefore be;

$$
N_{\text {Center }}^{\mathrm{RB}}=\left\lfloor\mathrm{CP}\left(T_{0}\right) \cdot N_{\text {Total }}^{\mathrm{RB}}\right\rfloor
$$

The resources in any sector $S_{i}$ can thus be found depending on the number of sector antennas and beamwidth as;

$$
N_{S_{i}}^{\mathrm{RB}}=\left\lfloor\frac{N_{\mathrm{Edge}}^{\mathrm{RB}}}{\left(2 \pi / \mathrm{d} \theta_{i}\right)}\right\rfloor
$$

Where $\mathrm{d} \theta_{i}$ represents the beamwidth of the sector $i$. Using the above resource allocation mechanism associates performance metrics with the FFR threshold $T_{0}$ allowing for design trade-offs in terms of load balancing, increasing peak rate or fairness similar to simulation-based grid models [6, 17-20].

\subsection{Network Sum Rate}

The overall average network sum rate can be derived from using previously derived expressions to be given as;

$$
\mathbb{T}_{\text {net }}=\frac{B \cdot N_{\mathrm{Edge}}^{\mathrm{RB}}}{\log _{e}(2)} \cdot \overline{\mathcal{T}}_{F F R_{\text {Edge }}}+\frac{B \cdot N_{\mathrm{Center}}^{\mathrm{RB}}}{\log _{e}(2)} \cdot \overline{\mathcal{T}}_{F F R_{\text {Center }}}
$$

$B$ is the bandwidth per resource block, $N_{\mathrm{Edge}}^{\mathrm{RB}}$ and $N_{\text {Center }}^{\mathrm{RB}}$ are given in (19) and (20) respectively, while the average UE rates are as earlier defined.

\section{Analytical and Numerical Results}

In this section, Monte-Carlo simulations are carried out in MATLAB and compared with our derived theoretical results for validation. A $20 \mathrm{MHz}$ bandwidth is considered with 100 available resource blocks, each with a bandwidth of $180 \mathrm{KHz}$. Macro base station transmit power $P_{T x, m}$ is given as $43 \mathrm{dBm}$ where $P_{T x, m}=1000 P_{f}$. The constant path loss is assumed to be $L_{0}=-34 \mathrm{~dB}$ (since shadowing effect is ignored) such that $P_{m}=P_{T X, m} \cdot L_{0}$. Practical values of outdoor path-loss exponents are used $(\alpha=3,4)$ in the analysis and thermal noise power $\sigma^{2}=-101 \mathrm{dBm}$. Macro base stations are modeled with density $\lambda_{m}=2.5$ base stations per $\mathrm{km}^{2}$ and $\lambda_{f}=4 \lambda_{m}$.

Sectored antennas are employed at the macro tier in 3 and 6 configuration for comparison purposes where the antennas are assumed to be uniformly aligned such that each sector of a MeNB is isolated from adjacent sectors by $2 \pi / \Delta$, where $\Delta$ is the number of sector antennas deployed in the edge corresponding to $\Delta$ edge FFR sub-bands. i.e. $\theta_{1}=[0,2 \pi / \Delta] \quad, \quad \theta_{2}=\theta_{1}+2 \pi / \Delta \quad, \quad \theta_{3}=\theta_{2}+2 \pi / \Delta \quad, \ldots$ $\theta_{\Delta}=\theta_{\Delta-1}+2 \pi / \Delta$. The gain pattern of the antennas is given in Table 2 .

Table 2. Antenna Gain Configuration.

\begin{tabular}{lll}
\hline Antenna Type & Antenna Pattern (in dB) & Parameter Values \\
\hline 3-Sectored Antenna & $G_{1}(\psi)=G_{\max }{ }^{*}-\min \left\{12\left(\frac{\psi-\pi}{\mathrm{HPBW}}\right)^{2}, A_{m}\right\}$ & $\begin{array}{l}G_{\text {max }}{ }^{*}=14 \mathrm{dBi}, \mathrm{HPBW}=70^{\circ} \quad A_{m}=23 \mathrm{~dB} \\
G_{\max }{ }^{*}=17 \mathrm{dBi}, \mathrm{HPBW}=35^{\circ} \quad A_{m}=20 \mathrm{~dB}\end{array}$ \\
\hline
\end{tabular}

Where $G_{\max }{ }^{*}$ is the bore-sight gain, $A_{m}$ is the maximum attenuation and HPBW is the Half-power beamwidth [30].

Since the random variable $\psi(x)$ is considered to be uniformly distributed over the range $[0,2 \pi)$, the CDF of $G_{m}(\psi)$ and $G_{m, \max }(\psi)$ can therefore be approximated as in (21) and (22) respectively;

$$
F_{G_{1}(\psi)}= \begin{cases}0 & \text { if } x<G_{\max }^{*}-A_{m} \\ 1-\frac{\mathrm{HPBW}}{\pi} \sqrt{\frac{G_{\max }{ }^{*}-x}{12}} & \text { if } G_{\max }{ }^{*}-A_{m} \leq x<G_{\max }{ }^{*} \\ 1 & \text { if } x \geq G_{\max }{ }^{*}\end{cases}
$$




$$
F_{G_{m, \max }(\psi)}= \begin{cases}0 & \text { if } x<G_{\max }{ }^{*}-12\left(\frac{\pi / \Delta}{\mathrm{HPBW}}\right) \\ 1-\frac{\Delta \times \mathrm{HPBW}}{\pi} \sqrt{\frac{G_{\text {max }}{ }^{*}-x}{12}} & \text { if } G_{\text {max }}{ }^{*}-12\left(\frac{\pi / \Delta}{\mathrm{HPBW}}\right) \leq x<G_{\max }{ }^{*} \\ 1 & \text { if } x \geq G_{\max }{ }^{*}\end{cases}
$$

These results can now be used in deriving coverage probability performance of edge UEs employing sectored FFR as defined in Lemma 3.

Figure 5 and Figure 6 show the coverage probability performance of a typical randomly located edge MUE under in the proposed heterogeneous network under closed access and employing 3-sectored FFR. The first observation is the close agreement between analytical and numerical results, with slight variations due to approximations and assumptions made in the theoretical model, thereby validating the proposed framework. Different values of the path-loss exponent $\alpha=4$ and $\alpha=2.5$ are used in both figures to investigate the difference between the case with noise and without noise. Due to higher attenuation of electromagnetic signals (in this case the interfering signals), the plots with $\alpha=4$ tend to show more variation between the results with noise and no-noise, while the plots with $\alpha=2.5$ are more interference limited. Going further, increasing the transmit power of the femtocells in Figure 6 leads to performance degradation in the coverage probability for both $\alpha=4$ and $\alpha=2.5$ plots. However, the latter experiences more severe degradation as can be seen due to weak attenuation of the increasing cross-tier interference.

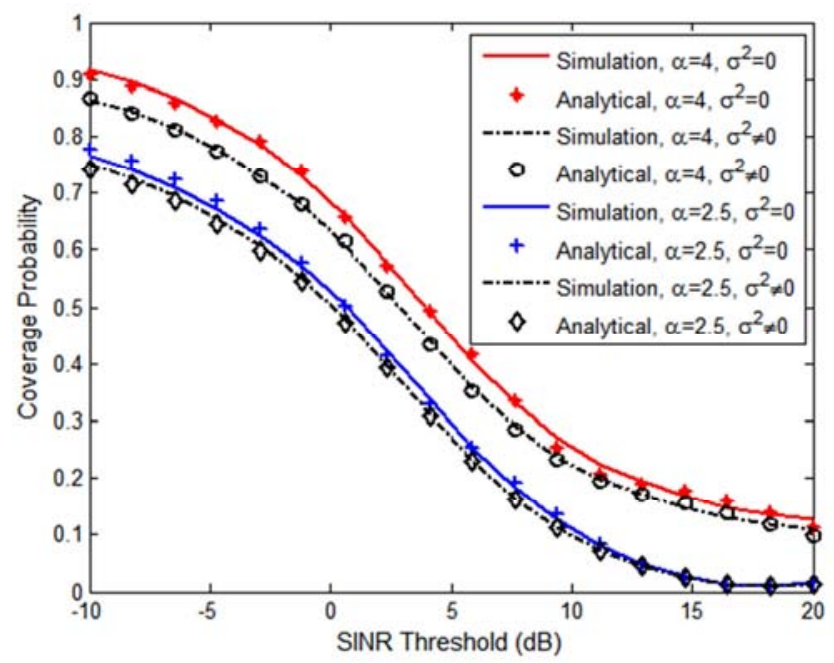

Figure 5. Coverage Probability for typical edge MUE under 3-sectored FFR in closed access. $T 0=3 \mathrm{~dB}, P_{T x, m}=1000 P_{f}, \lambda_{f}=4 \lambda_{m}$.

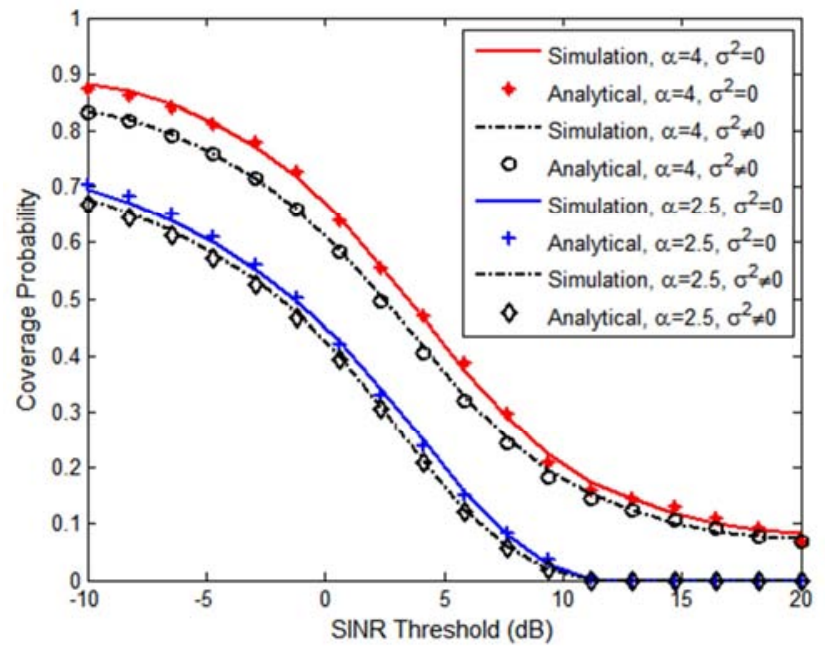

Figure 6. Coverage Probability for typical edge MUE under 3-sectored FFR in closed access $T_{0}=3 d B, P_{T x, m}=100 P_{f}, \lambda_{f}=4 \lambda_{m}$

Figure 7 then compares the coverage probability performance under the proposed sectored FFR schemes with traditional Strict, Soft and No-FFR schemes. Due to the narrow beamwidth of 6sectored antennas, interfering MeNB sectors are reduced by a factor of $1 / 6$ compared to $1 / 3$ for 3 -sectored antennas thereby yielding the best performance for the former. This point can be easily seen in Lemma 3 results where $\lambda_{m} \frac{\mathrm{d} \theta}{2 \pi}$ is used to thin the interfering macrocells for directional antennas. Also, in the sectored-FFR schemes, femtocells have higher number of subbands to choose from and so the probability of sharing same sub-bands with MUEs is reduced therefore further minimizing cross-tier interference. Strict FFR shows very good performance for low SINR thresholds, even comparable to 3-sectored FFR. This may be understood considering the fact that there are no interfering macrocells in the first tier under regular Strict FFR as shown in Figure 2(a) and even though for the irregular model used here it is not exactly true, the pattern of interfering cells will still be significantly reduced for the first tier as well.

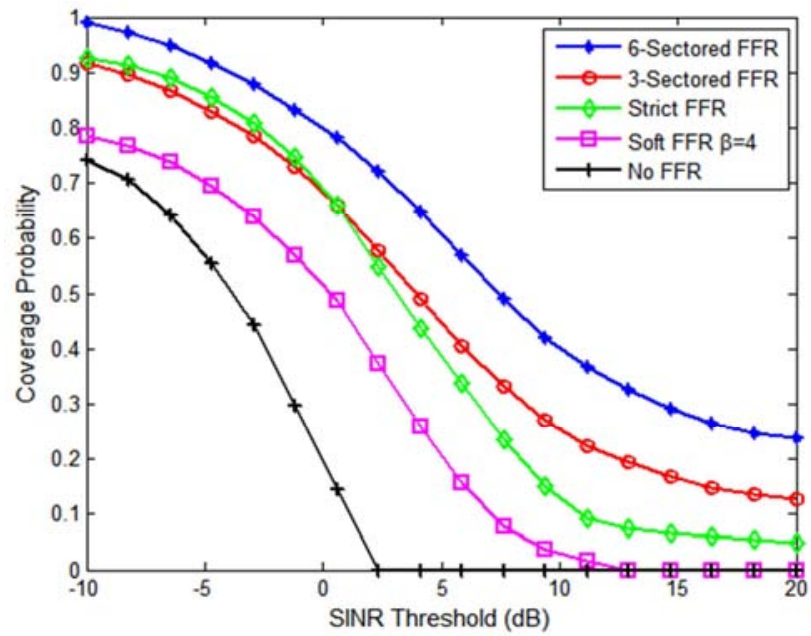

Figure 7. Comparing Coverage Probability performance under different FFR schemes in closed access. $T_{0}=3 d B, P_{T x, m}=1000 P_{f}$. 
The scheme with no FFR shows sharp drop in performance as the FFR threshold is approached since there are no sub-bands allocated when the instantaneous SINR of UEs falls below the threshold. These results therefore show that the proposed model is reasonably accurate for different system parameters such as high and low path-loss exponents, different femto transmit powers, FFR threshold and base station densities.

In Figure 8, the average rate for a typical edge MUE is plotted for different FFR schemes and the no-FFR scheme. Again, the results from Lemma 5 are validated for sectoredFFR with numerical simulations yielding the highest rates due to improved interference reduction and better resource utilization than other schemes. Increasing values of the FFR threshold correspond to edge UEs with relatively high SINR values already and hence the increasing trend of average rate with higher threshold values. Soft FFR can improve the coverage and rate performance of edge MUEs by use of higher power control factor as widely reported in literature hence we do not show this here, but at the cost of degraded performance to center UEs [6], [22], [24].

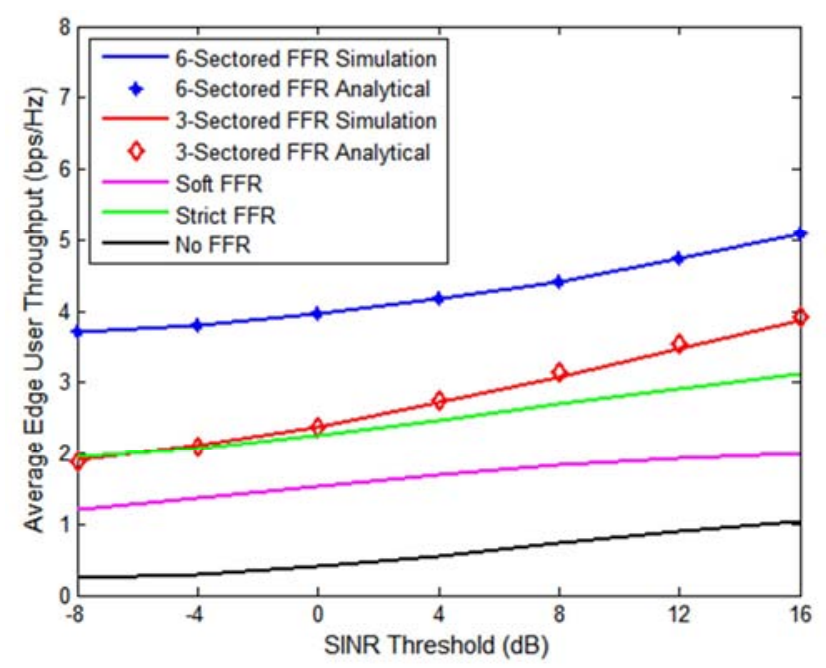

Figure 8. Average edge MUE rate under different FFR schemes.

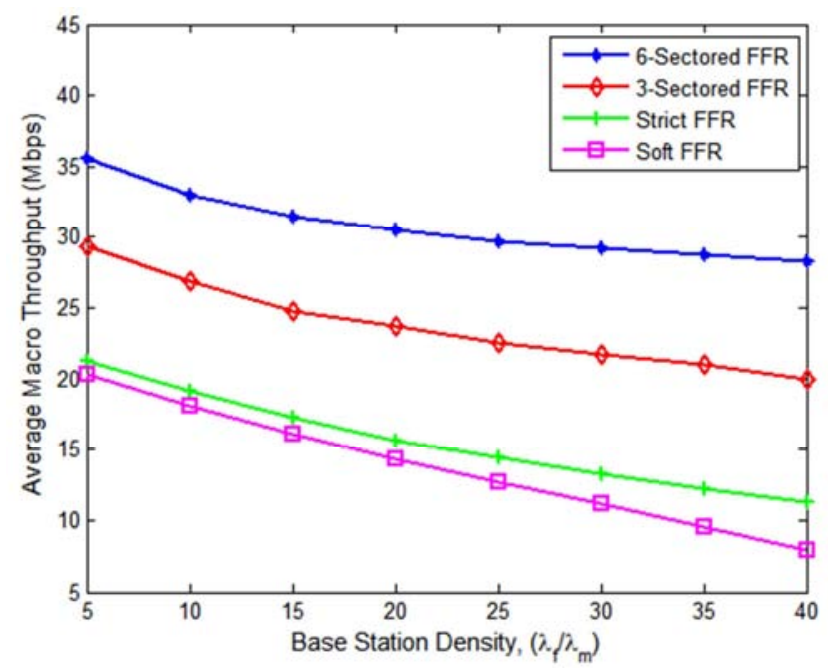

Figure 9. Average Macro tier Throughput with varying femtocell densities.
Lastly, the average macro tier network throughput is plotted in Figure 9 for varying femtocell densities, where there is an obvious reduction in network throughput as femtocell density increases for all schemes under closedaccess operation mode. The sectored FFR schemes show a little more tolerance to this effect due to multiple sub-bands available for the femtocells to select without necessarily interfering with the edge MUEs unlike other schemes. Soft FFR which inherently allows center and edge UEs to share same sub-bands exhibits the worst performance naturally.

\section{Conclusion}

In this paper, an analytical framework for coverage probability of a typically randomly located MUE employing sectored-FFR in two-tier macro-femto OFDMA network with closed access has been presented and validated by numerical simulations. A femtocell sensing algorithm minimizes crosstier interference by ensuring femtocells select sub-bands not in use by MUEs in close proximity, particularly at border of center and edge region which is usually ignored by many previous works. Two variants of the sectored FFR scheme are studied - 6-sectored and 3-sectored, and are compared to conventional FFR schemes and the no-FFR scheme in terms of coverage and rate performance. The sectored schemes showed better performance under different system configurations and varying network parameters, validating the model in both noise-limited and interference stricken cases. To the best of the author's knowledge, this is the first analytical framework using stochastic geometry to analyze sectored-FFR without violating the PPP properties through correlation or dependence.

An immediate extension of this work is to study the open access operation mode where off-loading is possible due to either load balancing or need for higher rates. The analysis will be more involved since the SINR distribution would depend not only on the macro tier FFR threshold but also the small cell's threshold value in order to be assigned an edge sub-band. Furthermore, more dynamic resource allocation schemes could be studied in such realistic, irregular deployments where allocations based on geographical size of coverage areas will not feasible. This contribution therefore presents a base-line platform for important, analytical modeling and analysis of practical Hetnets using FFR and sectorization.

\section{Acknowledgment}

This work was supported by the National Major Project under Grant No. 2015ZX03001013-002.

\section{Appendix A}

Proof of Lemma 1

Based on the ERPC model, the probability of coverage is defined as; 


$$
\begin{gathered}
\mathrm{CP}=\mathbb{P}[\mathrm{SINR}>T] \\
\int_{q>0} \mathbb{P}\left[\frac{P_{m} h_{m} r_{m}^{-\alpha}}{\sigma^{2}+I_{m}+I_{f}}>T \mid \widetilde{P}_{\text {max }}=q\right] f_{\widetilde{P}_{\max }}(q) \mathrm{d} q \\
\stackrel{\text { (a) }}{=} \int_{q>0} \mathbb{P}\left[h_{m}>\frac{\left(\sigma^{2} T+I_{m} T+I_{f} T\right)}{q} \mid \widetilde{P}_{\text {max }}=q\right] f_{\tilde{P}_{\text {max }}}(q) \mathrm{d} q \\
\stackrel{\text { (b) }}{=} \int_{q>0} e^{\left(-\frac{\sigma^{2} T}{q}\right)} e^{\left(-\frac{T}{q}\left(I_{m}+I_{f}\right)\right)} f_{\widetilde{P}_{\text {max }}}(q) d q \\
\stackrel{\text { (c) }}{=} \int_{q>0} e^{\left(-\frac{\sigma^{2} T}{q}\right)} \mathcal{L}_{I_{m}}\left(\frac{T}{q} \mid \widetilde{P}_{\text {max }}\right) \mathcal{L}_{I_{f}}\left(\frac{T}{q} \mid \widetilde{P}_{\text {max }}\right) f_{\widetilde{P}_{\text {max }}}(q) \mathrm{d} q
\end{gathered}
$$

Step (a) follows due to random variable $\widetilde{P}_{\max }$ as defined in (4).

Step (b) is as a result of Rayleigh fading where $h=\exp (1)$, and step (c) gives $\mathcal{L}_{I_{m}^{\wedge}}\left(\frac{T}{q} \mid \widetilde{P}_{\max }\right)$ and $\mathcal{L}_{I_{f}}\left(\frac{T}{q} \mid \widetilde{P}_{\max }\right)$, which are the Laplace transforms of the random variables $I_{m}$ and $I_{f}$ representing the interference experienced by the UE from all the macro BSs and femto BSs respectively.

First we find $\mathcal{L}_{I_{m}^{\wedge}}\left(\frac{T}{q} \mid \widetilde{P}_{\max }\right)$;

$$
\begin{aligned}
& \mathcal{L}_{I_{m}}\left(s \mid \widetilde{P}_{\max }\right)=\mathbb{E}_{I_{m}}\left[\exp \left(-s I_{m} \mid \widetilde{P}_{\max }\right)\right] \\
& \stackrel{\text { (a) }}{=} \mathbb{E}_{\phi_{k}}\left[\prod_{x \in \phi_{k} \backslash\left\{M_{0}\right\}} \mathbb{E}_{h_{x}}\left[\exp \left(-s P_{m} \cdot g_{x}\|x\|^{-\alpha}\right)\right] \mid \widetilde{P}_{\max }\right] \\
& =\mathbb{E}_{\phi_{k}}\left[\prod_{x \in \phi_{k} \backslash\left\{M_{0}\right\}} \frac{1}{1+s P_{m}\|x\|^{-\alpha} \mid} \widetilde{P}_{\max }\right] \\
& \stackrel{\text { (b) }}{=} \exp \left(-2 \pi \lambda_{m} \int_{\left(P_{m} / q\right)^{\frac{1}{\alpha}}}^{\infty}\left(1-\frac{1}{1+s P_{m} v^{-\alpha}}\right) v \mathrm{~d} v\right) \\
& \stackrel{(\text { c) }}{=} \exp \left(-2 \pi \lambda_{m} \int_{\left(P_{m} / q\right)^{\frac{1}{\alpha}}}^{\infty}\left(\frac{v^{\alpha}}{1+\frac{1}{(T / q) P_{m}}}\right)\right. \\
& \stackrel{(\mathrm{d})}{=} \exp \left(-2 \pi \lambda_{m} \int_{T^{-2 / \alpha}}^{\infty} \frac{1}{1+u^{\alpha / 2}} \cdot \frac{1}{2} \cdot\left((T / q) P_{m}\right)^{2 / \alpha} \mathrm{d} u\right) \\
& \stackrel{\text { (e) }}{=} \exp \left(-\pi \lambda_{m}\left(\frac{P_{m}}{q}\right)^{2 / \alpha} \rho(T, \alpha)\right)
\end{aligned}
$$

Where step (a) is from the i.i.d distribution of $\left\{g_{x}\right\}$ and step (b) is due to the PGFL of the PPP and Rayleigh fading assumption. Replacing $s=T / q$ and simplifying yields step (c). Using the relation $u=\frac{v^{2}}{\left(T P_{m} / q\right)^{2 / \alpha}}$ gives step (d) and upon simplification, the result is obtained in step (e) where
$\rho(T, \alpha)=\int_{T^{-2 / \alpha}}^{\infty} T^{2 / \alpha} \cdot \frac{1}{1+u^{\alpha / 2}} \cdot \mathrm{d} u$

Next, we find $\mathcal{L}_{I_{f}}\left(\frac{T}{q} \mid \widetilde{P}_{\max }\right)$, the femto tier interference component;

$$
\begin{aligned}
& \mathcal{L}_{I_{f}}\left(\mathrm{~s} \mid \widetilde{P}_{\max }\right) \stackrel{\text { (a) }}{=} E_{G_{f}, \Phi_{f}}\left[\exp \left[-s \sum_{x \in \Phi_{f}} P_{f} g_{f} R_{x}^{-\alpha}\right] \mid \widetilde{P}_{\max }\right] \\
& \stackrel{\text { (b) }}{=} \exp \left(-\lambda_{m} \int_{\mathrm{R}^{2}}\left(1-\mathcal{L}_{g}\left(s\|x\|^{-\alpha} P_{f}\right)\right) x \mathrm{~d} x\right) \\
& =\exp \left(-\lambda_{m} \int_{\mathrm{R}^{2}}\left(1-\frac{1}{1+P_{f}(T / q)\|x\|^{-\alpha}}\right) \mathrm{d} x\right) \\
& \mathcal{L}_{I_{f}}\left(\frac{T}{q} \mid \widetilde{P}_{\max }\right)=\exp \left(-\lambda_{m}\left(P_{f}(T / q)\right)^{2 / \alpha} \frac{2 \pi^{2}}{\alpha \sin \left(\frac{2 \pi}{\alpha}\right)}\right)
\end{aligned}
$$

Substituting $\mathcal{L}_{I_{m}^{\hat{m}}}\left(\frac{T}{q} \mid \widetilde{P}_{\text {max }}\right)$ from (A3 (e)), $\mathcal{L}_{I_{f}}\left(\frac{T}{q} \mid \widetilde{P}_{\text {max }}\right)$ from (A5) and $f_{\tilde{P}_{\max }}$ from (9) into (A2 (c)) gives the desired result of Lemma 1.

\section{Appendix B}

Proof of Lemma 3

For a typical MUE $y$, when $\operatorname{SINR}_{y}<T_{0}$ the UE is classified an edge UE and served by any one of $\Delta$ sectors with any of $\Delta$ edge sub-bands. The edge MUE now experiences new fading $\hat{g_{y}}$, and new co-tier and cross-tier interferences, $I_{m}^{\wedge}$ and $I_{f}^{\wedge}$, instead of $g_{y}, I_{m}$ and $I_{f}$. In deriving the CCDF of the edge UE, the previous SINR must be considered;

$$
\mathrm{CP}_{F F R-n}=\mathbb{P}\left(\frac{P_{m} G_{m, \max }\left(\psi_{1}(r)\right) h_{m}^{\wedge} r_{m}^{-\alpha}}{\sigma^{2}+I_{m}^{\wedge}+I_{f}^{\wedge}}>T \mid \frac{P_{m} h_{m} r_{m}^{-\alpha}}{\sigma^{2}+I_{m}+I_{f}}<T_{0}\right)
$$

Using Baye's rule,

$$
C P_{\{F F R-n\}}=\frac{\mathbb{P}\left(\frac{P_{m} G_{m, \text { max }}\left(\psi_{1}(r)\right) h_{m}^{\wedge} r_{m}^{-\alpha}}{\sigma^{2}+I_{m}^{\wedge}+I_{f}^{\wedge}}>T, \frac{P_{m} h_{m} r_{m}^{-\alpha}}{\sigma^{2}+I_{m}+I_{f}}<T_{0}\right)}{\mathbb{P}\left(\frac{P_{m} h_{m} r_{m}^{-\alpha}}{\sigma^{2}+I_{m}+I_{f}}<T_{0}\right)}
$$

Since $g_{y}$ and $g_{y}$ are i.i.d. exponentially distributed with unit mean;

$$
\frac{\mathbb{E}\left[\exp \left(-\frac{T}{q}\left(\sigma^{2}+I_{m}^{\wedge}+I_{f}^{\wedge}\right)\right)\left(1-\exp \left(-\frac{T_{0}}{q}\left(\sigma^{2}+I_{m}+I_{f}\right)\right)\right)\right]}{1-\mathbb{E}\left[\exp \left(-\frac{T_{0}}{q}\left(\sigma^{2}+I_{m}+I_{f}\right)\right)\right]}
$$


The first term of numerator represents $\mathrm{CP}\left(\left\{\lambda_{m}\right\},\left\{P_{m}\right\},\left\{T_{m}\right\},\left\{G_{m}().\right\}\right)$ as earlier derived, while the denominator is equivalent to the outage probability of a typical UE in the proposed model with omnidirectional antenna as defined by Lemma 1. i.e. denominator $=1-\mathrm{CP}_{\mathrm{cl}}\left(\left\{\lambda_{m}\right\},\left\{P_{m}\right\},\left\{T_{0}\right\}, 1\right)$. The second term of the numerator is resolved as given in (B4);

$$
=\mathbb{E}\left[\exp \left(-\frac{\sigma^{2}}{q}\left(T+T_{0}\right)\right) \cdot \exp \left(-\frac{\sigma^{2}}{q}\left(T I_{m}^{\wedge}+T_{0} I_{m}\right)\right) \cdot \exp \left(-\frac{1}{q}\left(T I_{f}^{\wedge}+T_{0} I_{f}\right)\right)\right]
$$

Second and third terms are the joint Laplace transforms for the random variables $I_{m}$ and $I_{m}^{\wedge}$, and $I_{f}$ and $I_{f}^{\wedge}$ respectively, which represent the interference from macro tier before and after FFR in the first case, and the interference from femto tier before and after FFR in the second case respectively.

$$
\begin{aligned}
& \mathcal{L}_{I_{m}, I_{m}}\left(\hat{s_{m}}, S_{m} \mid \widetilde{P}_{\max }\right)=\mathbb{E}\left[e^{\left(-\hat{\left.s_{m} I_{m}-s_{m} I_{m}\right)}\right.}\right] \\
& =\mathbb{E}\left[\exp \left(-\hat{s_{m}} \sum_{x \in \phi_{m \backslash\left\{M_{0}\right\}}} P_{m} G_{m}\left(\psi_{1}(x)\right) \cdot h_{x}^{\wedge}\|x\|^{-\alpha}-s_{m} \sum_{x \in \phi_{m \backslash\left\{M_{0}\right\}}} P_{m} h_{x}\|x\|^{-\alpha}\right)\right]
\end{aligned}
$$

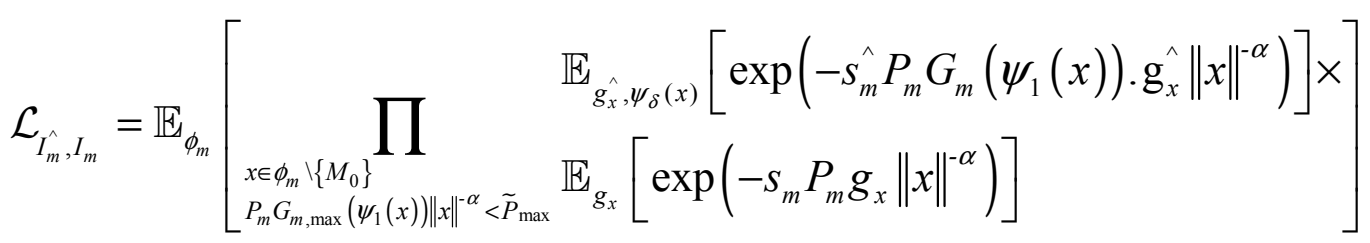

$$
\begin{aligned}
& \mathcal{L}_{I_{m}^{\wedge}, I_{m}}\left(\hat{s_{m}}, s_{m} \mid \widetilde{P}_{\max }\right)=\exp \left(-\frac{\lambda_{m}}{2}\left(\frac{P_{m}}{q}\right)^{2 / \alpha} M_{m}(T, \alpha)\right) \cdot \exp \left(-\pi \lambda_{m}\left(\frac{P_{m}}{q}\right)^{2 / \alpha} \rho(T, \alpha)\right) \\
& =\exp \left(-\lambda_{m}\left(\frac{P_{m}}{q}\right)^{2 / \alpha}\left(\frac{1}{2} M_{m}(T, \alpha)+\pi \rho(T, \alpha)\right)\right)
\end{aligned}
$$

Where $M(T, \alpha)=\int_{0}^{2 \pi}\left(G_{m, \text { max }}(\theta)\right)^{2 / \alpha} \rho\left(\frac{T G_{m}(\theta)}{G_{m, \text { max }}(\theta)}, \alpha\right) \mathrm{d} \theta$ The Joint Laplace transform for the femto-tier interference would be;

$$
\begin{gathered}
\mathcal{L}_{I_{f}^{\wedge}, I_{f}}\left(\hat{s_{f}}, s_{f} \mid \widetilde{P}_{\max }\right)=\mathbb{E}\left[\exp \left(-\hat{s_{f}} I_{f}^{\wedge}-s_{f} I_{f}\right)\right] \\
=\mathbb{E}\left[\exp \left(-\hat{s_{f}} \sum_{x \in \phi_{f}^{\hat{f}}} P_{f} g_{x}^{\wedge} R_{x}^{-\alpha}-s_{j} \sum_{x \in \phi_{f}} P_{f} g_{x} R_{x}^{-\alpha}\right)\right] \\
\mathcal{L}_{I_{f}^{\wedge}, I_{f}}\left(\hat{s_{f}}, s_{f} \mid \widetilde{P}_{\max }\right)=\exp \left(-\pi\left(\frac{P_{f}}{q}\right)^{2 / \alpha} \frac{2 \pi}{\alpha \sin (2 \pi / \alpha)}\left(T^{2 / \alpha} \lambda_{f}^{\wedge}+T_{0}^{2 / \alpha} \lambda_{f}\right)\right)
\end{gathered}
$$

After finding all contents of second term of numerator, now de-conditioning on the maximum equivalent power $\widetilde{P}_{\text {max }}$ gives the expression for $\mathcal{Z}(k, T)$ given in (B10). Substituting all above results in (B3) gives the desired result of Lemma 3.

$$
\mathcal{Z}(k, T)=\left[\begin{array}{l}
\left.\exp \left(-\frac{\sigma^{2}}{q}\left(T+T_{0}\right)\right) \cdot \exp \left(-\lambda_{m}\left(\frac{P_{m}}{q}\right)^{2 / \alpha}\left(\frac{1}{2} M_{m}(T, \alpha)+\pi \rho\left(T_{0}, \alpha\right)\right)\right) \cdot\right] \\
\exp \left(-\pi\left(\frac{P_{f}}{q}\right)^{2 / \alpha} \frac{2 \pi}{\alpha \sin (2 \pi / \alpha)}\left(T \lambda_{f}^{\wedge}+T_{0} \lambda_{f}\right)\right)
\end{array}\right] \cdot f_{\tilde{P}_{\text {max }}}(q) \mathrm{dq}
$$




\section{Appendix C}

Proof of Lemma 5

The average rate of a typical UE averaged over the base station locations and fading distribution expressed in units of nats $/ \mathrm{Hz}$ is defined as

$$
\overline{\mathcal{T}}=\mathbb{E}[\ln (1+\mathrm{SINR})]=\int_{q>0} \mathbb{E}[\ln (1+\operatorname{SINR})] \cdot f_{\widetilde{P}_{\max }}(q) \mathrm{dq}
$$

Since $\overline{\mathcal{T}}$ is a positive random variable, $\mathbb{E}[\mathcal{T}]=\int_{t>0} P(\mathcal{T}>t) \mathrm{dt}$

When using sectored-FFR, we condition the new SINR of the UE on the previous one which was less than the FFR threshold to be defined as edge UE. Hence we write the rate as;

$$
\overline{\mathcal{T}}_{F F R}=\int_{q>0} \int_{t>0} \mathbb{P}[(*)] \mathrm{dt} . f_{\widetilde{P}_{\max }}(q) \mathrm{dq}
$$

Where $\mathbb{P}[(*)]$ is simply evaluated as in Lemma 1 .

$$
\mathbb{P}[(*)]=\frac{\mathbb{P}\left[\ln \left(1+\frac{P_{m} G_{m, \max }\left(\psi_{1}(r)\right) h_{m}^{\wedge} r_{m}^{-\alpha}}{\sigma^{2}+I_{m}^{\wedge}+I_{f}^{\prime}}\right)>t, \frac{P_{m} h_{m} r_{m}{ }^{-\alpha}}{\sigma^{2}+I_{m}+I_{f}}<T_{0}\right]}{\mathbb{P}\left[\frac{P_{m} h_{m} r_{m}^{-\alpha}}{\sigma^{2}+I_{m}+I_{f}}<T_{0}\right]}
$$

Following similar derivations as before yields the final result;

$$
\overline{\mathcal{T}}_{F F R_{\text {Edge }}}=\int\left[\frac{\mathrm{CP}_{\left(\left\{\lambda_{m}\right\},\left\{P_{m}\right\},\left\{e^{t}-1\right\},\left\{G_{m}(.)\right\}\right)}-\int_{0}^{\infty} \mathcal{R} \cdot f_{\tilde{P}_{\max }}(q) \mathrm{d} q}{1-\mathrm{CP}_{\left.\mathrm{cl} .\left\{\lambda_{m}\right\},\left\{P_{m}\right\},\left\{T_{0}\right\}, 1\right)}}\right] \mathrm{dt}
$$

Where

$$
\begin{aligned}
& \mathcal{R}=\int_{0}^{\infty}\left[\exp \left(-\frac{\sigma^{2}}{q}\left(e^{t}-1+T_{0}\right)\right) \cdot \mathcal{L}_{I_{m}^{\hat{m}}, I_{m}}\left(\hat{s_{m}}, s_{m} \mid \widetilde{P}_{\max }\right) \cdot \mathcal{L}_{I_{f}^{\hat{f}}, I_{f}}\left(\hat{s_{f}}, s_{f} \mid \widetilde{P}_{\max }\right)\right] \\
& \text { And } \hat{s_{m / f}}=\frac{e^{t}-1}{q} \text { and } s_{m / f}=\frac{T_{0}}{q} .
\end{aligned}
$$

The final result is therefore realized in $(\mathrm{C} 4)$ similar to Lemma 5 defined in (18).

\section{References}

[1] D. L. P. Xiaolu Chu, Yang Yang, Fredrik Gunnarasson, Heterogeneous Cellular Networks Theory, Simulation and Deployment. New York, United States of America: Cambridge University press, 2013.

[2] N. Saquib, E. Hossain, L. Long Bao, and K. Dong In, "Interference management in OFDMA femtocell networks: issues and approaches," Wireless Communications, IEEE, vol. 19, pp. 86-95, 2012.

[3] T. Zahir, K. Arshad, A. Nakata, and K. Moessner, "Interference management in femtocells," IEEE Communications Surveys \&
Tutorials, vol. 15, pp. 293-311, 2013.

[4] V. Chandrasekhar, M. Kountouris, and J. G. Andrews, "Coverage in multi-antenna two-tier networks," IEEE Transactions on Wireless Communications, vol. 8, pp. 53145327, 2009.

[5] V. Chandrasekhar and J. G. Andrews, "Spectrum allocation in tiered cellular networks," Communications, IEEE Transactions on, vol. 57, pp. 3059-3068, 2009.

[6] N. Saquib, E. Hossain, and K. Dong In, "Fractional frequency reuse for interference management in LTE-advanced hetnets," Wireless Communications, IEEE, vol. 20, pp. 113-122, 2013.

[7] S. Mukherjee, Analytical Modeling of Heterogeneous Cellular Networks: Geometry, Coverage, and Capacity: Cambridge University Press, 2014.

[8] M. Haenggi, Stochastic Geometry for Wireless Networks. New York: Cambridge University Press, 2013.

[9] J. G. Andrews, F. Baccelli, and R. K. Ganti, "A Tractable Approach to Coverage and Rate in Cellular Networks," IEEE Transactions on Communications, vol. 59, pp. 3122-3134, 2011.

[10] H. S. Dhillon, R. K. Ganti, F. Baccelli, and J. G. Andrews, "Modeling and Analysis of K-Tier Downlink Heterogeneous Cellular Networks," IEEE Journal on Selected Areas in Communications, vol. 30, pp. 550-560, 2012.

[11] R. W. Heath, M. Kountouris, and T. Bai, "Modeling heterogeneous network interference using Poisson point processes," IEEE Transactions on Signal Processing, vol. 61, pp. 4114-4126, 2013.

[12] H. Wang and M. C. Reed, "A novel tractable framework to analyse heterogeneous cellular networks," in 2011 IEEE GLOBECOM Workshops (GC Wkshps), 2011, pp. 287-292.

[13] M. Haenggi, J. G. Andrews, F. Baccelli, O. Dousse, and M. Franceschetti, "Stochastic geometry and random graphs for the analysis and design of wireless networks," IEEE Journal on Selected Areas in Communications, vol. 27, pp. 1029-1046, 2009.

[14] H. ElSawy, E. Hossain, and M. Haenggi, "Stochastic Geometry for Modeling, Analysis, and Design of Multi-Tier and Cognitive Cellular Wireless Networks: A Survey," IEEE Communications Surveys \& Tutorials, vol. 15, pp. 996-1019, 2013.

[15] E. Dinc and M. Koca, "On dynamic fractional frequency reuse for OFDMA cellular networks," in Personal Indoor and Mobile Radio Communications (PIMRC), 2013 IEEE 24th International Symposium on, 2013, pp. 2388-2392.

[16] O. G. Aliu, M. Mehta, M. A. Imran, A. Karandikar, and B. Evans, "A New Cellular-Automata-Based Fractional Frequency Reuse Scheme," IEEE Transactions on Vehicular Technology, vol. 64, pp. 1535-1547, 2015.

[17] A. S. Mohamed, M. Abd-Elnaby, and S. A. El-Dolil, "Selforganised dynamic resource allocation scheme using enhanced fractional frequency reuse in long term evolution-advanced relay-based networks," IET Communications, vol. 10, pp. 1163-1174, 2016.

[18] D. Bilios, C. Bouras, V. Kokkinos, A. Papazois, and G. Tseliou, "Selecting the optimal fractional frequency reuse scheme in long term evolution networks," Wireless personal communications, vol. 71, pp. 2693-2712, 2013. 
[19] H. Kalbkhani, V. Solouk, and M. G. Shayesteh, "Resource allocation in integrated femto-macrocell networks based on location awareness," Communications, IET, vol. 9, pp. 917932, 2015.

[20] C. Y. Oh, M. Y. Chung, H. Choo, and T.-J. Lee, "Resource allocation with partitioning criterion for macro-femto overlay cellular networks with fractional frequency reuse," Wireless personal communications, vol. 68, pp. 417-432, 2013.

[21] S. U. Abdullahi, L. Jian, H. Ci, and Z. Xiaonan, "Enhancing throughput performance in LTE-Advanced Hetnets with buffered Fractional Frequency Reuse," in 2016 Eighth International Conference on Ubiquitous and Future Networks (ICUFN), 2016, pp. 918-923.

[22] T. D. Novlan, R. K. Ganti, J. G. Andrews, and A. Ghosh, "A New Model for Coverage with Fractional Frequency Reuse in OFDMA Cellular Networks," in 2011 IEEE Global Telecommunications Conference - GLOBECOM 2011, 2011, pp. 1-5.

[23] T. D. Novlan, R. K. Ganti, A. Ghosh, and J. G. Andrews, "Analytical Evaluation of Fractional Frequency Reuse for OFDMA Cellular Networks," IEEE Transactions on Wireless Communications, vol. 10, pp. 4294-4305, 2011.

[24] T. D. Novlan, R. K. Ganti, and J. G. Andrews, "Coverage in Two-Tier Cellular Networks with Fractional Frequency Reuse," in 2011 IEEE Global Telecommunications Conference - GLOBECOM 2011, 2011, pp. 1-5.
[25] T. D. Novlan, R. K. Ganti, A. Ghosh, and J. G. Andrews, "Analytical Evaluation of Fractional Frequency Reuse for Heterogeneous Cellular Networks," Communications, IEEE Transactions on, vol. 60, pp. 2029-2039, 2012.

[26] P. Mitran and C. Rosenberg, "On fractional frequency reuse in imperfect cellular grids," in 2012 IEEE Wireless Communications and Networking Conference (WCNC), 2012, pp. 2967-2972.

[27] R. Ullah, N. Fisal, H. Safdar, Z. Khalid, and W. Maqbool, "Fractional frequency reuse for irregular geometry based heterogeneous cellular networks," in Information Technology: Towards New Smart World (NSITNSW), 2015 5th National Symposium on, 2015, pp. 1-6.

[28] H. Wang and M. C. Reed, "Tractable model for heterogeneous cellular networks with directional antennas," in 2012 Australian Communications Theory Workshop (AusCTW), 2012, pp. 61-65.

[29] F. o. B. a. B. Błaszczyszyn, Stochastic Geometry and Wireless Networks, Volum I: Theory vol. Voluma I: Now Publishers Inc., 2009.

[30] S. Kumar, I. Z. Kovacs, G. Monghal, K. I. Pedersen, and P. E. Mogensen, "Performance Evaluation of 6-Sector-Site Deployment for Downlink UTRAN Long Term Evolution," in Vehicular Technology Conference, 2008. VTC 2008-Fall. IEEE 68th, 2008, pp. 1-5. 